Research Article

\title{
Extended Spectrum Beta-Lactamase Producing Enterobacteriaceae: Hospital-Acquired Urinary Tract Infections, Khartoum-Sudan
}

\author{
Omnia M Hamid ${ }^{1 *}$, Samia A Gumaa ${ }^{2}$, Al Amin Ibrahim ${ }^{3}$ and Magdi Bayoumi ${ }^{4}$ \\ ${ }^{1}$ Faculty of Medical Laboratory Sciences, Department of Medical Microbiology, University of Medical Sciences \& \\ Technology, Sudan \\ ${ }^{2}$ Professor of Microbiology, Head of Microbiology Department, Royal Care International Hospital (RCIH), Sudan \\ ${ }^{3}$ Associate Professor of Medical Microbiology, Dean of the Faculty of Medical Laboratory Sciences, University of \\ Khartoum, Sudan \\ ${ }^{4}$ Associate Professor of Pathology, Director, Ibrahim El Zain Training Centre (IBRAZ), Ibn Sina University, Khartoum \\ Sudan
}

\begin{abstract}
Background: The family Enterobacteriaceae are the most common causative agent of urinary tract infection (UTI) pathogen among both hospital and community patients. Antibiotic resistance among uropathogens has become a major public health problem that due to easy resistant spread and transfer resistant gene within gram negative bacteria, also might led to limitations of treatment options. This study was aimed to determine the prevalence of Extended Spectrum ß-Lactamase (ESBL)-producing Enterobacteriaceae isolates from urine samples in patients admitted in Academy Charity Teaching Hospital (ACTH) by phenotypic detection, Khartoum State, Sudan.
\end{abstract}

Methods: A descriptive; cross-sectional study was conducted on 350 Clean-catch, mid-stream urine samples (MSU) from both gender (female and male) patients hospitalized in Academic Charity Teaching Hospital with sign and symptom of UTI. The gram-negative isolates were identified and screened by Kirby-Bauer disc diffusion method. Double-Disc Synergy Test (DDST) was performed for detection and confirmation of ESBLs. Socio-demographic and clinical data were obtained from each participant using close-end questionnaires with palace to sign or stamp (a way to protect patients confidentiality) and check list for practical follow-up work and record.

Results: A total of $104 \mathrm{MSU}$ samples were diagnosed to have growth of Enterobacteriaceae species for significant bacteriuria ( $\geq 10^{5} / \mathrm{CFU}$ ); Escherichia coli was the most frequently isolated organism (43.3\%); followed by Klebsiella pneumoniae (22.1\%), Proteus mirabilis (11.5\%), Enterobacter aerogenes (7.7\%), Klebsiella oxytoca and Seratia marcescens (5.8\%) for each and (1.9\%) of Enterobacter cloacae and Citrobacter freundii for each. ESBL was detected in 67 (64.4\%) isolates including $82.2 \%(37 / 45)$ of $E$. coli, $91.3 \%(21 / 23)$ of $K$. pneumonia resistant to cefotaxime $(30 \mu \mathrm{g})$ in screening ESBL detection. Double-disk synergy test (DDST); ESBL confirmatory method was performed on Enterobacteriaceae isolated species $23.1 \%(24 / 104)$ was ESBL producing uropathogens, which included 13 isolates of $E$. coli $(28.9 \%)$ and 8 of $K$. pneumoniae (34.8\%).

Conclusions: The high prevalence of ESBL producing Enterobacteriaceae among hospitalized patients with UTI, indicates to develop a monitoring system of the drug resistance for scientific-based selection of antibiotic therapy and requires hospital policies on empirical use of antibiotic. Routine ESBL phenotypic detection testing and subsequent antibiogram with disk diffusion method could be perform as easy and costless to third countries.

\section{Keywords}

Extended-spectrum ß-lactamases-Urinary tract infection, Enterobacteriaceae

\begin{abstract}
Abbreviations
MSU: Mid-Stream Urine; HAI: Hospital Acquired Infection; GNB: Gram Negative Bacteria; ESBL: Extended Spectrum BetaLactamase; NCCLS: National Committee on Clinical Laboratory Standards; CLSI: Clinical Laboratory Standards Institute; UTIs: Urinary Tract Infections; MHA: Mueller Hinton Agar; MDR: Multi Drug Resistance; CFU: Colony Forming Unit; E.coli: Escherichia coli; K. pneumoniae: Klebsiella pneumoniae; DDST: Double Disk Synergy Test; E. colaceae: Enterobacter cloacae; E. aerogenes: Enterobacter aerogenes; K. oxytoca: Klebsiella oxytoca; P. mirabilis: Proteus mirabilis; C. freundii: Citrobacter freundii; S. marcescens: Serratia marcescens; spp: species
\end{abstract}

Copyright: (c) 2019 Hamid OM, et al. This is an open-access article distributed under the terms of the Creative Commons Attribution License, which permits unrestricted use, distribution, and reproduction in any medium, provided the original author and source are credited. 
Citation: Hamid OM, Gumaa SA, Ibrahim AA, et al. (2019) Extended Spectrum Beta-Lactamase Producing Enterobacteriaceae: HospitalAcquired Urinary Tract Infections, Khartoum-Sudan. Ann Microbiol Res 3(1):76-82

\section{Background}

Gram-negative bacteria (GNB) mainly Enterobacteriaceae family are predominate in hospital-acquired urinary tract infections; urinary tract infection (UTI) is one of the most common GNB infections acquired either from community or hospital settings and affecting all age groups, sexes and might asymptomatic or symptomatic depending on the pathogenesis of infection [1]. Also, most of bacteriuria cases are generally asymptomatic, and an effective management is removing the catheter rather than antibiotic treatment. Worldwide; It is the most common nonsurgical hospital acquired infection (HAI) and the second most common healthcare associated infection which constitute $40-50 \%$ of all hospital infections also as the second most common reason for empirical antibiotic treatment, UTI is a major driver of antibiotic usage globally [2].

Multi-drug resistance GNB are common challenge to patient-hospital safety and protection control due to limit treatment options [3]. These Enterobacteriaceae family strains have an efficient mechanism by up-regulating or acquiring genes that code for drug resistance [2], production of $\beta$-lactamases is the primary mechanism of $\beta$-lactam resistance in these pathogens [4]. Extended spectrum $\beta$-lactamase (ESBL) can confer resistance to penicillins, first-, second-, and thirdgeneration cephalosporins, and aztreonam; they are usually hindered by $\beta$-lactamase inhibitors such as clavulanic acid. Most ESBLs can also hydrolyze fourth-generation cephalosporins (e.g., cefepime or cefpirome). Furthermore, Enterobacteriacea $e$ harboring ESBLs are often co-resistant to fluoroquinolones, aminoglycosides, and trimethoprim/sulfamethoxazole [5]. In Gram negative bacteria these enzymes remain in the periplasmic space, where they attack the antibiotic before it can reach its receptor site. ESBL is the first plasmid mediated $\beta$-lactamase was described in early 1960 mainly among Enterobacteriaceae family and other GNB $[6,7]$. The emergence of resistance to extended-spectrum cephalosporins remains challenging; these agents are often first-line therapy [8,9]. Unfortunately, the plasmids carrying ESBL genes often carry resistance determinants targeting fluoroquinolones as well. Where the sensitivity and specificity in traditional susceptibility tests are unable to detect ESBLs; has led to the search for an accurate and cost-effective test to detect the presence of ESBL in hospitalized patients. The aim of this study was to determine the prevalence of ESBL producing hospital Enterobacteriaceae in urinary tract infection.

\section{Methods}

\section{Study design}

A facility-based descriptive cross-sectional study was conducted. The research was conducted during the period of December 2007 to July 2008 in the urology department of Academy Charity Teaching Hospital (ACTH) located in Khartoum State, Sudan. All females and males participants, aged 20 to 65 years, admitted in the urology department of ACTH for UTI were included in the study. Were excluded patients with primary negative urine culture and whose urine culture grew negative for Enterobacteriaceae, day-care patients, pregnant women, diabetic and patients hospitalized for other underline infection not related UTI.

\section{Collection of samples}

A total of clean-catch midstream urine (MSU) samples ( $\mathrm{n}$ $=350)$ were collected, from males $(n=157)$ and females $(n=$ 193 ) in-patients with a mean age of 40 years diagnosed with recurrent urinary tract infection, in sterile and screw capped disposable containers. Patients aged $<18$ years considered as pediatric population and having specific treatment guidelines, were excluded. Specimens such as a nephrostomy, ileal conduit, extra prostatic secretion, suprapubic aspirate, or bag specimens were also excluded as they represent distinct clinical scenarios. Well defined guidance was provided to patients to avoid contamination of urine samples. All the collected specimens were properly labeled, and data were recorded through a standardized questionnaire.

\section{Isolation and identification of bacterial species}

Uncentrifuged urine samples were processed by calibrated loop onto blood agar and MacConkey agar plates, immediately or within 2 hours from collection. Plates were incubated aerobically at $37^{\circ} \mathrm{C}$ ( 24 hours) overnight; the cut-off value of $\geq 10^{5} \mathrm{CFU} / \mathrm{mL}$ was considered as significant growth [10]. Himedia culture and biochemical's test media, and API 20E (bioMerieux, Marcy-l'Etoile, France) for biochemical confirmations were used according to the manufacturer's instructions.

\section{Antimicrobial susceptibility test}

For screening ESBL production the antimicrobial susceptibility test was performed using Kirby-Bauer disc diffusion technique as recommended by the Clinical and Laboratory Standards Institute [11] (CLSI, 2011). Mueller-Hinton agar culture medium (Himedia, India) was inoculated by a direct saline suspension of isolated colonies with turbidity of $0.5 \mathrm{Mc}$ Farland. Then antibiotic disks (Oxoid, UK), cefotaxime (30 $\mu \mathrm{g})$, ceftazidime $(30 \mu \mathrm{g})$, cefpodoxime $10 \mu \mathrm{g}$ and aztreonam $30 \mu \mathrm{g}$ were placed on the agar surface at a distance of $30 \mathrm{~mm}$ (center to center) from each other. After 18 hours of incubation at $37{ }^{\circ} \mathrm{C}$, results were interpreted by measuring the inhibition zone diameter around each disk. According to CLSI criteria, an inhibition zone of $\leq 27 \mathrm{~mm}$ for aztreonam and $\leq 22 \mathrm{~mm}$ for ceftazidime, cefotaxime and cefpodoxime indicated that the strain possibly produced ESBL. E. coli ATCC ${ }^{\circledR} 25922$ was used as control strains for both.

\section{Confirmatory test for ESBL production}

The Double-Disc Synergy Test (DDST) by Jarlier [12] was performed for all isolates resistant to at least two of applied

*Corresponding author: Omnia M Hamid, Faculty of Medical Laboratory sciences, Department of Medical Microbiology, University of Medical Sciences \& Technology, Khartoum-Sudan 11111, Sudan, Tel: +249912678663

Accepted: June 22, 2019

Published online: June 24, 2019

Citation: Hamid OM, Gumaa SA, Ibrahim AA, et al. (2019) Extended Spectrum Beta-Lactamase Producing Enterobacteriaceae: Hospital-Acquired Urinary Tract Infections, Khartoum. Ann Microbiol Res 3(1):76-82 
Citation: Hamid OM, Gumaa SA, Ibrahim AA, et al. (2019) Extended Spectrum Beta-Lactamase Producing Enterobacteriaceae: HospitalAcquired Urinary Tract Infections, Khartoum-Sudan. Ann Microbiol Res 3(1):76-82

Table 1: Distribution of significant and no significant growth of Enterobacteriaceae from inpatients urine samples.

\begin{tabular}{|c|c|c|c|c|c|c|c|c|}
\hline \multirow{3}{*}{ Gender } & \multirow{3}{*}{$\begin{array}{l}\text { Number (\%) of } \\
\text { urine samples }\end{array}$} & \multicolumn{2}{|c|}{ Enterobacteriaceae isolates } & \multicolumn{3}{|c|}{ Odd ratio } & \multirow{2}{*}{$\begin{array}{l}\text { Pearson Chi- } \\
\text { square value }\end{array}$} & \multirow{3}{*}{$P$ value } \\
\hline & & \multirow{2}{*}{$\begin{array}{l}\text { No significant growth } \\
\left(<10^{5} \mathrm{cfu} / \mathrm{mL}\right)\end{array}$} & \multirow{2}{*}{$\begin{array}{l}\text { Significant growth } \\
\left(>10^{5} \mathrm{cfu} / \mathrm{mL}\right)\end{array}$} & \multirow{2}{*}{ Value } & \multicolumn{2}{|l|}{$95 \% \mathrm{Cl}$} & & \\
\hline & & & & & Lower & upper & \multirow{4}{*}{$1.5822 ; \mathrm{df}=2$} & \\
\hline Female & 193 (55.14\%) & $141-73.06 \%$ & $52-26.94 \%$ & \multirow{3}{*}{0.74} & \multirow{3}{*}{0.47} & \multirow{3}{*}{1.17} & & \multirow{3}{*}{$0.4533^{*}$} \\
\hline Male & $157(44.86 \%)$ & $105-66.87 \%$ & $52-33.12 \%$ & & & & & \\
\hline Total & $350(100.00 \%)$ & $246-70.29 \%$ & $104-29.71 \%$ & & & & & \\
\hline
\end{tabular}

\%: Percentage; Cl: Confidence interval; df: Degree of freedom; ${ }^{*}$ Insignificant.

Table 2: Frequency and distribution of Enterobacteriaceae isolates from urine samples $(n=104)$.

\begin{tabular}{|l|l|}
\hline Bacterial isolates & Number (\%) \\
\hline E.coli & $45(43.3 \%)$ \\
\hline K. pneumonia & $23(22.1 \%)$ \\
\hline P. mirabilis & $12(11.5 \%)$ \\
\hline E. aerogenes & $8(7.7 \%)$ \\
\hline K. oxytoca & $6(5.8 \%)$ \\
\hline S. marcescens & $6(5.8 \%)$ \\
\hline E. cloacae & $2(1.9 \%)$ \\
\hline C. freundii & $2(1.9 \%)$ \\
\hline n (\%) & $\mathbf{1 0 4}(\mathbf{2 9 . 7 \% )}$ \\
\hline
\end{tabular}

Keynote: $\mathrm{n}=$ total number of isolates.

antibiotics in that are used in the screening method to confirm the ESBL production. Mueller-Hinton agar (Himedia, India) was inoculated with saline suspension of isolated microorganism adjusted to 0.5 McFarland turbidity standards, and DDST was performed by placing disks of ceftazidime, cefotaxime, aztreonam (30 $\mathrm{g}$, each) and cefpodoxime (10 $\mu \mathrm{g})$ at a distance of $20 \mathrm{~mm}$ (center to center) from a disk of amoxicillin/clavulanic acid $(20 \mu \mathrm{g} / 10 \mu \mathrm{g})$. After $18-20$ hours of incubation at $35 \pm 2{ }^{\circ} \mathrm{C}$. Positive result is indicated when the inhibition zones around any of the $3^{\text {rd }}$ generation cephalosporin discs are augmented in the direction of the disc containing clavulanic acid.

\section{Statistical analysis}

The data collected were computerized through Microsoft Excel and analyzed through statistical package for social sciences version 16.0 (SPSS). Univariate analysis was performed, and data was summarized in frequency tables to estimate prevalence. Probability values of $<0.05$ were considered as statistically significant for all results analysis by chi-square test.

\section{Results}

Three hundred fifty urine samples were collected from hospitalized patients at long stay ward ( $>4-<26$ days); most of the patients had signs and symptoms of UTI under pathologist recommendation. And 72 out of 350 were already covered with antibiotic empirical dose. The prevalence of UTI due to Enterobacteriaceae among female inpatients were $55.14 \%$ $(n=193)$ and the remaining $44.86 \%(n=157)$ were males, their age ranged from 20 to 65 years, with average of 40 years \pm 11.64 . Where 104 urine samples had growth of Enterobacteriaceae equally distributed in gender; there was no significant difference in the gender and Enterobacteriaceae growth between significant and non-significant UTI among inpatients selections with $p$-value 0.4533 (Table 1 ). The predominating pathogen was $E$. coli $43.3 \%(n=45)$, followed by $K$. pneumoniae $22.1 \%(n=23)$. Table 2; summarize the distribution of the various Enterobacteriaceae species isolated.

Of 104 isolated Enterobacteriaceae Species, 24 (23.1\%) were ESBL-positive which included 13 isolates of $E$. coli $(54.2 \%), 8$ isolates of $K$. pneumoniae (33.3\%) and 3 isolates of other species (4.2\%).

\section{Antimicrobial susceptibility testing of the iso- lates}

The susceptibility pattern of isolated ESBL-producing Enterobacteriaceae species, determined by disk diffusion test revealed that isolates with higher resistant $64.4 \%$ (67/104) were towards cefotaxime and cefpodoxime had lowest resistance $32.7 \%(34 / 104)$ of isolates. Cefpodoxime was the most effective antibiotics on E. coli and K. pneumoniae and other species tested. On the other hand, Enterobacteriaceae strains showed high resistance to cefotaxime antibiotics, which could be used as clue antibiotic for ESBL screening test (Table 3).

\section{Confirmatory tests for ESBL detection}

The Double-Disc Synergy Test (DDST) by Jarlier [12] was observed for cefotaxime with Amoxicillin/clavulanic acid against the isolates, 24 (23.1\%) of Enterobacteriaceae isolates were ESBL-positive to CTX/AMX, CAZ/AMX 18 (17.3\%), AZT/ AMX 15 (14.4\%) and Low ESBL production was detected using SPD/AMX 6 (5.8\%). The DDDT method it is significant with $p$ value 0.000 at $p<0.05$ and chi-square statistic is 32.8251, which indicated that the cefotaxime disc it is the better disc use for confirmation (Table 4).

\section{Discussion}

As urinary tract infection is commonest disease among hospitalized patients among both gender, its diagnosis and treatment have important consequences for patients' health, development of antibiotics resistance, spreading of multidrug resistant strains and health care costs [13]. Among hospitalized patients with UTIs at this public (ACTH), teaching hospital in Khartoum-Sudan, the prevalence of UTI due to Enterobacteriaceae was 104 (29.7\%) out of 350 patients, 
Citation: Hamid OM, Gumaa SA, Ibrahim AA, et al. (2019) Extended Spectrum Beta-Lactamase Producing Enterobacteriaceae: HospitalAcquired Urinary Tract Infections, Khartoum-Sudan. Ann Microbiol Res 3(1):76-82

Table 3: $3^{\text {rd }}$ generation cephalosporins antibiotic resistance pattern of isolated Enterobacteriaceae determined by disk diffusion (ESBL screening) test.

\begin{tabular}{|c|c|c|c|c|}
\hline \multirow{2}{*}{ Bacterial isolates } & Cefotaxime & Aztreonam & Ceftazidime & Cefpodoxime \\
\hline & Resistant, n (\%) & Resistant, n (\%) & Resistant, n (\%) & Resistant, n (\%) \\
\hline E. coli $(n=45)$ & $37(82.2 \%)$ & 38 (84.4\%) & $30(66.7 \%)$ & $16(35.6 \%)$ \\
\hline K. pneumoniae $(\mathrm{n}=23)$ & $21(91.3 \%)$ & 17 (73.9\%) & $19(82.6 \%)$ & 13 (56.5\%) \\
\hline P. mirabilis $(\mathrm{n}=12)$ & $4(33.3 \%)$ & $3(25 \%)$ & $1(8.3 \%)$ & $\mathbf{0}(0 \%)$ \\
\hline E. aerogenes $(n=8)$ & $1(12.5 \%)$ & $3(37.5 \%)$ & $2(25 \%)$ & $2(25 \%)$ \\
\hline K. oxytoca $(\mathrm{n}=6)$ & $0(0 \%)$ & $2(33.3 \%)$ & $0(0 \%)$ & $1(16.7 \%)$ \\
\hline S. marcescens $(n=6)$ & $2(33.3 \%)$ & $2(33.3 \%)$ & $3(50 \%)$ & $1(16.7 \%)$ \\
\hline E. cloacae $(\mathrm{n}=2)$ & $1(50 \%)$ & $0(0 \%)$ & $1(50 \%)$ & $0(0 \%)$ \\
\hline C. freundii $(\mathrm{n}=2)$ & $1(50 \%)$ & $1(50 \%)$ & $1(50 \%)$ & $1(50 \%)$ \\
\hline Total $(n=104)$ & 67 (64.4\%) & 66 (63.5\%) & 57 (54.8\%) & 34 (32.7\%) \\
\hline
\end{tabular}

${ }^{*} 3^{\text {rd }} \mathrm{GC}$ : Third generation cephalosporins.

Table 4: Results of DDST method for isolated ESBL-producing Enterobacteriaceae resistant to $3^{\text {rd }}$ GC antibiotics in ESBL screening test.

\begin{tabular}{|c|c|c|c|c|}
\hline Number (\%) & CTX/AMX & CAZ/AMX & AZT/AMX & CPD/AMX \\
\hline Bacterial isolates & ESBL, n (\%) & ESBL, n (\%) & ESBL, n (\%) & ESBL, n (\%) \\
\hline E. coli $(\mathrm{n}=45)$ & $13(28.9 \%)$ & $9(20 \%)$ & $6(13.3 \%)$ & 2 (4.4\%) \\
\hline K. pneumoniae $(\mathrm{n}=23)$ & $8(34.8 \%)$ & $4(17.4 \%)$ & $6(26.1 \%)$ & $4(17.4 \%)$ \\
\hline P. mirabilis $(\mathrm{n}=12)$ & $0(0 \%)$ & $0(0 \%)$ & $0(0 \%)$ & $0(0 \%)$ \\
\hline E. aerogenes $(n=8)$ & $1(12.5 \%)$ & $2(25 \%)$ & $1(12.5 \%)$ & $0(0 \%)$ \\
\hline K. oxytoca $(\mathrm{n}=6)$ & $1(16.7 \%)$ & $1(16.7 \%)$ & $1(16.7 \%)$ & $0(0 \%)$ \\
\hline S. marcescens $(n=6)$ & $1(16.7 \%)$ & $2(33.3 \%)$ & $1(16.7 \%)$ & $0(0 \%)$ \\
\hline E. cloacae $(\mathrm{n}=2)$ & $0(0 \%)$ & $0(0 \%)$ & $0(0 \%)$ & $0(0 \%)$ \\
\hline C. freundii $(\mathrm{n}=2)$ & $0(0 \%)$ & $0(0 \%)$ & $0(0 \%)$ & $0(0 \%)$ \\
\hline Total & 24 (23.1\%) & $18(17.3 \%)$ & 15 (14.4\%) & 6 (5.8\%) \\
\hline
\end{tabular}

${ }^{*}$ CAZ: Ceftazidime; CPD: Cefpodoxime; AZT: Aztreonam; CTX: Cefotaxime; AMX: Co-amoxiclav disc.

this prevalence is similar to the published data in Chicago (29\%) [14]; elsewhere, higher prevalence of UTI due to Enterobacteriaceae was reported in Saudi Arabia (73.5\%) [15] and Nigeria (81.7\%) [16]. The differences observed between the studies can be due to the methods and resistance patterns could be related to environmental factors and antimicrobial susceptibility test used. In this study, the number of male patients to female patient's ratio was 1:1.2. And among positive cases, the ratio was 1:1.27. Females were higher in both cases which was natural enrolment bias. Females are more frequently affected due to colonization of urethra with colonic Gram-negative bacteria because of its proximity to anus and short length of urethra as patient's gender is risk factor of UTI [17].

Antimicrobial resistance has become a serious worldwide public health issue. Infections caused by drug resistant bacteria are responsible for increased morbidity, mortality, prolong hospital stay and increased government hospital costs. The prevalence of Enterobacteriaceae ESBL producing was (23.1\%) in our research, as appeared in Khartoum 2007 that give us alarming at that time to search on prevalence of ESBL producer. A recent study in Sudan 2014 [18] reported 65\%, higher than our 2008 findings. The increase may be related to the lack of guidelines for treatment of UTI, and the misuse of antibiotics. The prevalence of ESBL producing Enterobacteriaceae varies geographically worldwide from $(<1 \%$ to $74 \%)$ [19]. It is reported as (10\%) [20] in East Europe, (3.5\%) [21] in a Canadian study and (20-48.8\%) [22] in Asia. Within the Arabian Gulf region, various ESBL prevalence were reported (7.5\%) [23] in Kuwait, (41.0\%) [24] in United Arab Emirates and (22.0\%) [25] in Saudi Arabia. In the African region [26] Algeria, Egypt and Nigeria reported $31.4 \%, 19.0 \%$, and $12.8 \%$ hospital acquired ESBL producing Enterobacteriaceae respectively. These findings impose the need of suitable use of antibiotics based on accurate bacteriological testing along with appropriate guidelines.

Increasing unreasonable and miss-use of antibiotics, sales of substandard antibiotics and transmission of drug resistant bacteria among hospitalized patients may be responsible for the rise in antibiotic resistance among the bacteria. By screening method 67 (64.4\%) Enterobacteriaceae species isolates were found to be resistant to cefotaxime, followed by aztreonam $63.5 \%$, ceftazidime $54.8 \%$ and low resistant was $32.7 \%$ to Cefpodoxime. This finding is lower than those obtained by Kamlesh K [27] who reported cefotaxime (74.74\%) and ceftazidime (83.16\%). Empirical antibiotic selection should be 
Citation: Hamid OM, Gumaa SA, Ibrahim AA, et al. (2019) Extended Spectrum Beta-Lactamase Producing Enterobacteriaceae: HospitalAcquired Urinary Tract Infections, Khartoum-Sudan. Ann Microbiol Res 3(1):76-82

based on knowledge of the local prevalence of bacterial organisms and antibiotic sensitivities, because resistance patterns may vary according to the region of concern.

On the other hand $82.6 \%$ of $E$. coli were resistant to CTX and $91.3 \%$ of $K$. pneumoniae ESBL positive isolates in the screening test from urine in-patients was the most common ESBL producer, This percentages is considered high when compared to published studies in India; as described in 2005 [28] were $82.6 \%$ of $K$. pneumoniae, and $66.7 \%$ of $E$. coli and $19.8 \%$ E. coli and $31.8 \%$ K. pneumoniae resistant to CAZ and in 2008 [29]. Lower prevalence's were reported elsewhere in Korea (4.8\%), Taiwan (8.5\%) and Hong Kong (12.0\%) [3032]. However, E. cloacae (20 ESBL positive isolates) had a high prevalence than other classic producers (33.3\% of $P$. mirabilis were resistant to CTX $\& 50 \%$ of $S$. marcescens was resistant to CAZ). The same observation was made in 2008 [29].

The ubiquity of ESBL-producing $E$. coli was observed highlighting the importance of regular surveillance of ESBL producing clinical isolates in clinical samples to minimize increasing of multi-drug resistance strains among hospital and community, to prevent the ineffectiveness of antimicrobial agent for good health practices [33]. Our study revealed that the ESBLs producing uropathogenic $E$. coli isolates were $28.9 \%$. This finding is almost the same as the study reported $(26.87 \%) E$. coli by Kamlesh [27]; our results are slightly higher than data published by various authors such as [34-36] who reported ESBLs producer $24.5 \%, 19.02 \%$ and $21.4 \%$ respectively. Other authors reported higher prevalence of $E$. coli ESBL producer of $61.2 \%, 35 \%$ from Suadia Arabia [25] and 42.31\% Khartoum [18] respectively. The variability of the reported prevalence of UTI with ESBL producing Enterobacteriaceae in inpatients may be related to both the antibiotic and the microorganism tested across countries.

ESBL-producing $K$. pneumoniae as an important urinary pathogen in this group of patients. However, K. pneumoniae also seems to represent an important urinary pathogen in young females as supported by $34.8 \%$ is the high isolation rates in this group of patients observed in our study and by This result is in line with findings reported in a previous study in Italy [37]. Conversely, the high rates for non - ESBL mediated cefpodoxime resistant Enterobacteriaceae isolates $5.8 \%$ may be due to their different mechanisms for resistance. This further limits the therapeutic options available to treat these infections.

The ESBLs-producing Enterobacteriaceae were most frequent in older age group in our study; also associated with risk factors such as prolonged hospitalization, previous antibiotic use, and presence of invasive devices [38]. According to the majority of isolates were from patient's age between 40 to 70 years, as our findings revealed a high level of the ESBL-producing Enterobacteriaceae in hospital acquired UTIs in patients of aged $>40$ years, other studies also pointed that ESBL isolates are encountered more frequently in the elderly $[38,39]$.

The high resistant to antibiotic in our community may be due to empirical use of drug from patients relatives plus easy to get medication from pharmacist, and mainly most of the older inpatients used traditional and herbal medication that might be one of the risk factors that play a major role in develop antimicrobial resistant not only to cephalosporin also extend to other antimicrobial type and in 2008 there are no studies showing a rate of ESBL producing Enterobacteriaceae in Khartoum state which gave us highlighting to search at that time according resistant observation among Enterobacteriaceae during daily hospital report.

\section{Conclusion}

At that time 2008 this is the first study conducted to determine the prevalence of Enterobacteriaceae producing ESBL among hospitalized patients with UTI, with the effect of gender and age on its prevalence, and highlight to screen ESBL with DDDT method which is easy to perform and cost less to our community as a routine antimicrobial susceptibility test in hospitals microbiology laboratories, at least for antibioticresistant microorganisms, for best practice in treatments of UTI. ESBL producing Enterobacteriaceae was observed in $23.1 \%$ of the total isolates.

\section{Conflict of Interest Statement}

We declare that we have no conflict of interest.

\section{Acknowledgments}

A deep appreciation goes to Dr. Mounkaila Noma for helping us throughout paper editing \& formatting.

\section{Funding}

The research was fully funded by the author in the frame of a master degree submitted to the Faculty of Medical Laboratory Sciences at the University of Medical Sciences and Technology. No funding source.

\section{Availability of Data and Materials}

All relevant data is reported in the manuscript and attached document upon request.

\section{Authors Contributions}

Omnia M Hamid developed the study design, collected, processed samples, analyzed samples and prepared (M.Sc research degree), Samia A Gumma thesis supervisor, and Al Amin Ibrahim was samples process and lab test technique reviewer, Magdi Bayoumi approved the final thesis and manuscript writing.

\section{Ethical Consideration}

Well verbal informed the participant; participation was voluntary and anytime had the right to withdraw from the study, and the questionnaire and consent form of the participants agreement was either to sign or stamp (because some patients uneducated) on the research tool on compilation of the data collection, process or published. The research project was approved by the research committee (SUMASRI-international Review Board); Faculty of Medical Laboratory Sciences, University of Medical Sciences and Technology, Khartoum-Sudan. 
Citation: Hamid OM, Gumaa SA, Ibrahim AA, et al. (2019) Extended Spectrum Beta-Lactamase Producing Enterobacteriaceae: HospitalAcquired Urinary Tract Infections, Khartoum-Sudan. Ann Microbiol Res 3(1):76-82

\section{Competing Interests} ests.

The authors declare that they have no competing inter-

\section{Consent for Publication}

All authors read and approved to the submitted version of this manuscript.

\section{References}

1. Galatti L, Sessa A, Mazzaglia G, et al. (2006) Antibiotic prescribing for acute and recurrent cystitis in primary care: A 4 year descriptive study. Journal of Antimicrobial Chemotherapy 57: 551-556.

2. Savas L, Guvel S, Onlen Y, et al. (2006) Nosocomial urinary tract infections: Micro-organisms, antibiotic sensitivities and risk factors. West Indian Med J 55: 188-193.

3. Klevens RM, Edwards JR, Richards Jr CL, et al. (2007) Estimating health care-associated infections and deaths in US hospitals, 2002. Public Health Rep 122: 160-166.

4. Andrade SS, Sader HS, Jones RN, et al. (2006) Increased resistance to first-line agents among bacterial pathogens isolated from urinary tract infections in Latin America: Time for local guidelines? Mem Inst Oswaldo Cruz 101: 741-748.

5. Paterson DL, Bonomo RA (2005) Extended-spectrum $\beta$-lactamases: A clinical update. Clin Microbiol Rev 18: 657-686.

6. Datta N, Kontomichalou P (1965) Penicillinase synthesis controlled by infectious $R$ factors in Enterobacteriaceae. Nature 208: 239-241.

7. Bradford PA (2001) Extended-spectrum $\beta$-lactamases in the $21^{\text {st }}$ century: Characterization, epidemiology, and detection of this important resistance threat. Clin Microbiol Rev 14: 933-951.

8. Jacobsen Sá, Stickler D, Mobley H, et al. (2008) Complicated catheter-associated urinary tract infections due to Escherichia coli and Proteus mirabilis. Clin Microbiol Rev 21: 26-59.

9. Nicolas-Chanoine M-H, Blanco J, Leflon-Guibout V, et al. (2008) Intercontinental emergence of Escherichia coli clone O25: H4ST131 producing CTX-M-15. J Antimicrob Chemother 61: 273-281.

10. Cheesbrough M (2006) District laboratory practice in tropical countries. Cambridge university press.

11. Wayne $P$ (2007) Clinical and laboratory standards institute. Performance standards for antimicrobial susceptibility testing 17.

12. Jarlier V, Nicolas M-H, Fournier G, et al. (1988) Extended broad-spectrum $\beta$-lactamases conferring transferable resistance to newer $\beta$-lactam agents in Enterobacteriaceae: hospital prevalence and susceptibility patterns. Rev Infect Dis 10: 867-878.

13. Magliano E, Grazioli V, Deflorio L, et al. (2012) Gender and age-dependent etiology of community-acquired urinary tract infections. ScientificWorldJournal 2012.

14. Khawcharoenporn T, Vasoo S, Singh K (2013) Urinary tract infections due to multidrug-resistant enterobacteriaceae: Prevalence and risk factors in a Chicago emergency department. Emerg Med Int 2013.

15. Abdel-Fattah MM (2005) Surveillance of nosocomial infections at a Saudi Arabian military hospital for a one-year period. Ger Med Sci 3

16. Otajevwo $F(2013)$ Urinary tract infection among symptomatic outpatients visiting a tertiary hospital based in midwestern Nigeria. Glob J Health Sci 5: 187.

17. Forbes BA, Sahm DF, Weissfeld AS (2007) Study guide for Bailey \& Scott's diagnostic microbiology. Mosby USA.

18. Almugadam BS, Ibrahim KA, Ali MA (2016) Phenotypic detection of Extended Spectrum ßeta-Lactamase producing Escherichia coli isolated from urinary tract infected Patients in Khartoum, Sudan.

19. Thomson K, Prevan A, Sanders C (1996) Novel plasmid-mediated beta-lactamases in enterobacteriaceae: emerging problems for new beta-lactam antibiotics. Curr Clin Top Infect Dis 16: 151-163.

20. Coque T, Baquero F, Canton R (2008) Increasing prevalence of ESBL-producing Enterobacteriaceae in Europe. Euro Surveill 13: 19044.

21. Zhanel GG, DeCorby M, Laing N, et al. (2008) Antimicrobialresistant pathogens in intensive care units in Canada: results of the Canadian National Intensive Care Unit (CAN-ICU) study, 2005-2006. Antimicrob Agents Chemother 52: 1430-1437.

22. Jones RN (1999) Summation: Beta-lactam resistance surveillance in the Asia-Western Pacific region. Diagn Microbiol Infect Dis 35: 333-338.

23. Jamal W, Rotimi V, Khodakhast F, et al. (2005) Prevalence of extended-spectrum beta-lactamases in Enterobacteriaceae, Pseudomonas and Stenotrophomonas as determined by the VITEK 2 and E test systems in a Kuwait teaching hospital. Med Princ Pract 14: 325-331.

24. Al-Zarouni M, Senok A, Rashid F, et al. (2008) Prevalence and antimicrobial susceptibility pattern of extended-spectrum betalactamase-producing Enterobacteriaceae in the United Arab Emirates. Med Princ Pract 17: 32-36.

25. Kandeel AY (2014) Prevalence and risk factors of extendedspectrum B-lactamases producing Enterobacteriaceae in a general hospital in Saudi Arabia. Journal of Microbiology and Infectious Diseases 4: 50-54.

26. Storberg V (2014) ESBL-producing Enterobacteriaceae in Africa-a non-systematic literature review of research published 20082012. Infect Ecol Epidemiol 4: 20342.

27. Yadav KK, Adhikari N, Khadka R, et al. (2015) Multidrug resistant Enterobacteriaceae and extended spectrum $\beta$-lactamase producing Escherichia coli: a cross-sectional study in National Kidney Center, Nepal. Antimicrob Resist Infect Control 4: 42.

28. Duttaroy B, Mehta S (2005) Extended spectrum b lactamases (ESBL) in clinical isolates of Klebsiella pneumoniae and Escherichia coli. Indian J Pathol Microbiol 48: 45-48.

29. Shahid M, Malik A, Akram M, et al. (2008) Prevalent phenotypes and antibiotic resistance in Escherichia coli and Klebsiella pneumoniae at an Indian tertiary care hospital: plasmidmediated cefoxitin resistance. Int J Infect Dis 12: 256-264.

30. Hanberger $H$, Diekema D, Fluit A, et al. (2001) Surveillance of antibiotic resistance in European ICUs. J Hosp Infect 48: 161-176.

31. Ho P, Tsang D, Que T, et al. (2000) Comparison of screening methods for detection of extended-spectrum $\beta$-lactamases and their prevalence among Escherichia coli and Klebsiella species in Hong Kong. APMIS 108: 237-240.

32. Pai H, Lyu S, Lee JH, et al. (1999) Survey of extended-spectrum $\beta$-lactamases in clinical isolates of Escherichia coli and Klebsiella pneumoniae: Prevalence of TEM-52 in Korea. J Clin Microbiol 37: 1758-1763. 
Citation: Hamid OM, Gumaa SA, Ibrahim AA, et al. (2019) Extended Spectrum Beta-Lactamase Producing Enterobacteriaceae: HospitalAcquired Urinary Tract Infections, Khartoum-Sudan. Ann Microbiol Res 3(1):76-82

33. Chakrawarti A, Dongol P, Khanal H, et al. (2015) Extended Spectrum Beta Lactamases Detection and Multiple Antibiotic Resistance Indexing of Escherichia Coli from Urine Samples of Patients from a Referral Hospital of Eastern Nepal. International Journal of Applied Sciences and Biotechnology 3: 423-426.

34. Ibrahim ME, Bilal NE, Magzoub MA, et al. (2013) Prevalence of extended-spectrum $\beta$-lactamases-producing Escherichia coli from Hospitals in Khartoum State, Sudan. Oman Med J 28: 116-120.

35. Mobaleghi J, Salimizand H, Beiranvand S, et al. (2012) Extended spectrum B-lactamases in urinary isolates of escherichia coli in five Iranian hospitals. Asian Journal of Pharmaceutical and Clinical Research 5: 35-36.

36. Datta P, Gupta V, Sidhu S (2014) Extended spectrum beta lactamase positive uropathogenic E. coli epidemiological factors and resistance. BJMP 7: 718

37. Spanu T, Luzzaro F, Perilli M, et al. (2002) Occurrence of extended-spectrum $\beta$-lactamases in members of the family Enterobacteriaceae in Italy: Implications for resistance to $\beta$-lactams and other antimicrobial drugs. Antimicrob Agents Chemother 46: 196-202.

38. Mumtaz S, Ahmad M, Aftab I, et al. (2007) Extended spectrum $\beta$-lactamases in enteric gram-negative bacilli: related to age and gender. J Ayub Med Coll Abbottabad 19: 107-111.

39. Roshan M, Ikram A, Mirza IA, et al. (2011) Susceptibility pattern of extended spectrum ß-lactamase producing isolates in various clinical specimens. J Coll Physicians Surg Pak 21: 342-634. 